\title{
The Evolution of Metastatic Colorectal Cancer Clinical Trials: Application of the ASCO Framework for Assessing Value
}

\author{
Doreen A. Ezeife, MD, FRCPC ${ }^{\text {a }}$ S Sunil Parimi, MD, FRCPC ${ }^{\text {b }}$ Ellen R. Cusano, BSc ${ }^{c}$; \\ Matthew K. Smith, BSc ${ }^{\text {; }}$; Tony H. Truong, MD, MPH, FRCPC ; Soundouss Raissouni, MD; \\ Yongtao Lin, MLIS'; Jose G. Monzon, MD, PhD, FRCPC ${ }^{\text {h }}$; Haocheng Li, PhD ; \\ Vincent C. Tam, MD, FRCPC ${ }^{\text {h }}$; and Patricia A. Tang, MD, FRCPC ${ }^{\text {h }}$
}

\begin{abstract}
Background: Phase III trials in metastatic colorectal cancer (mCRC) have collectively led to progressive advancements in patient outcomes over the past decades. This study characterizes the evolution of $\mathrm{mCRC}$ phase III trials through assessing the value of cancer therapy, as measured by the ASCO Value Framework. Methods: Phase III trial results of systemic therapy for mCRC published between 1980 and 2015 were identified, and their outcome, statistical significance, journal impact factor, and citation by the 2016 NCCN Clinical Practice Guidelines in Oncology (NCCN Guidelines) for CRC were recorded. For each trial, the net health benefit (NHB) score was calculated using the June 2015 (original) and May 2016 (revised) ASCO Value Framework: Advanced Disease. Results: There were 114 mCRC phase III trials eligible for calculation of the NHB score. Using the revised framework, the median NHB score was 4.6 (range, -30 to 43.5 ); $12 \%$ of trials received bonus points. Trials with statistically significant results had higher NHB scores compared with nonsignificant trials (median NHB score, 21.6 vs 2.9; $P<.0001$ ). Clinical trials cited in the NCCN Guidelines had higher NHB scores than those not cited (median score, 8.0 vs $0.3 ; P=.02$ ). In multivariate linear regression analysis, the only significant predictor of high NHB score was statistically significant studies. Conclusions: The median NHB score for $\mathrm{mCRC}$ phase III trials was 4.6. Higher NHB scores are associated with statistically significant studies and are cited in the NCCN Guidelines, a surrogate for practice-changing trials. The 2016 ASCO Value Framework may not fully capture the benefits on an individual patient level.
\end{abstract}

\section{Background}

Systemic therapy for metastatic colorectal cancer (mCRC) has evolved over the past 20 years to incorporate inhibitors of angiogenesis and the epidermal growth factor receptor, with or without cytotoxic che-

From aPrincess Margaret Cancer Centre, University Health Network, Toronto, Ontario; 'british Columbia Cancer Centre, Vancouver Island Centre, Victoria, British Columbia; 'University of Ottawa, Ottawa, Ontario; dUniversity of Calgary, Calgary, Alberta; 'Alberta Children's Hospital, University of Calgary, Calgary, Alberta; ${ }^{\prime}$ Margery E. Yuill Cancer Centre, Medicine Hat, Alberta; ${ }^{9}$ Alberta Health Services, Calgary, Alberta; and h'Tom Baker Cancer Centre, University of Calgary, Calgary, Alberta, Canada. Submitted December 21, 2016; accepted for publication April 12, 2017. Dr. Parimi has disclosed that he has served on advisory boards for Janssen, Astellas, Pfizer, Amgen, and Novartis. The remaining authors have disclosed that they have no financial interests, arrangements, affiliations, or commercial interests with the manufacturers of any products discussed motherapy. This therapeutic armamentarium of potential treatment sequences has contributed to substantial improvements in survival. For example, the median overall survival (mOS) of patients with KRAS wild-type $\mathrm{mCRC}$ randomized to cetuximab and FOLFIRI in the

in this article or their competitors.

Author contributions: Principal investigator: Tang. Search strategy: Lin. Study concept and design: Tam, Tang. Database generation: Parimi, Raissouni, Lin, Monzon. Data acquisition: Ezeife, Parimi, Cusano, Smith, Raissouni, Monzon, Tam. Data analysis and interpretation: Ezeife, Cusano, Smith, Truong, Li, Tam. Manuscript preparation: Ezeife. Critical revision: Parimi, Cusano, Smith, Truong, Raissouni, Lin, Monzon, Li, Tam, Tang. Guarantor of work and final approval: all authors.

Correspondence: Doreen A. Ezeife, MD, FRCPC, Princess Margaret Cancer Centre, University Health Network, 610 University Avenue, Toronto, Ontario, Canada M5G 2M9. E-mail: doreen.ezeife@uhn.ca 
FIRE-3 trial was 33 months $^{1}$; this represents a near doubling of the mOS reported by the pivotal trials that established the addition of irinotecan to fluorouracil and leucovorin in 2000. 2,3 These advancements have shifted how we perceive the effectiveness of mCRC therapies. In the metastatic setting, the value of a new cancer therapeutic encompasses the benefits of treatment on OS or progression-free survival (PFS), the impact on quality of life (QoL), and the economic cost and toxicity. ${ }^{4}$ For instance, the addition of panitumumab to combination chemotherapy yielded a 4-month OS improvement in the PRIME clinical trial. ${ }^{5}$ However, this targeted therapy costs approximately $\$ 6,300$ USD per month and is accompanied by cutaneous and other side effects. Value is a dynamic concept and its definition may change depending on the tumor site, clinical indication, point in time, cost, and specific patient interests. $^{6}$

In June 2015, ASCO published a value framework that incorporated measures of clinical benefit, toxicity, and palliation of patient symptoms to generate net health benefit (NHB) scores. ${ }^{7}$ The "net" in NHB is determined by comparing a given drug to its comparator-control, often in a phase III randomized clinical trial setting. This NHB score is weighed against the drug's cost to determine the overall value of a new cancer treatment. ASCO updated its value framework in May 2016 to incorporate additional elements, such as QoL. ${ }^{8}$ This study evaluated the utility of the ASCO Value Framework by systematically applying the framework to mCRC clinical trials to provide a concrete clinical context. This study also characterized the evolution of phase III clinical trials in mCRC over the past 3 decades, with respect to NHB score, using the ASCO Value Framework.

\section{Methods}

A systematic search for pertinent articles reporting phase III clinical trials of systemic therapy for mCRC conducted between 1980 and November 2015 were performed in Ovid MEDLINE (see supplemental eAppendix 1, available with this article at JNCCN. org). Relevant articles were also hand-searched on PubMed. Interim or repeat analyses of original papers were excluded. Any trial that did not focus solely on the effectiveness of systemic therapy was excluded. Only large phase III trials with at least
100 patients per arm were included. For trials that involved multiple (ie, >2) arms, the control arm and the investigational arm with the most efficacious primary end point were compared. As per the ASCO Value Framework, clinical trials that did not demonstrate statistical significance in the clinical trial end points analyzed were included.

For each phase III trial published between 1980 and November 2015, the NHB score was calculated using both the original June 2015 and the revised May 2016 ASCO Value Framework: Advanced Disease. Three blinded reviewers (D.A.E., M.K.S., E.R.C.) independently calculated the NHB score of each clinical trial; if there was disagreement, a consensus was reached to determine a final score. A trial can obtain a negative NHB score if outcomes are superior in the control arm compared with the experimental arm. The revised 2016 framework awards bonus points for a $50 \%$ improvement in the proportion of patients alive in the experimental regimen at the time point on the survival curve that is double the mOS or PFS of the control arm (tail of the curve).$^{8}$ Awarding tail-of-the-curve bonus points was determined by visually analyzing the appropriate survival curve. In addition, journal impact factor and citation by the 2016 NCCN Clinical Practice Guidelines in Oncology (NCCN Guidelines) for Colon and Rectal Cancers, Versions 1 and 2 (the most upto-date, widely used mCRC guidelines available), was determined. ${ }^{9,10}$ For each trial, average mOS was determined by dividing the sum of the mOS reported by the number of arms in the trial. The incremental cost and incremental cost-effectiveness ratio (ICER) of available regimens were extracted from the Canadian Agency for Drugs and Technologies in Health pan-Canadian Oncology Drug Review (CADTH pCODR) Web site. ${ }^{11}$ Cost calculation includes costs for drug acquisition, molecular testing, resources, and surgical resection (eg, liver resection). ${ }^{11}$

Statistical analysis involved comparison of the 2016 NHB scores to line of therapy, trial sample size, journal year of publication, impact factor, and citation by the 2016 NCCN Guidelines for CRC to identify associations. Original and revised ASCO Value Framework: Advanced Disease NHB scores were also compared to determine correlations. Comparisons were made using chi-square and Fisher exact test for categorical data. Kruskal-Wallis and $t$ tests were used to compare continuous factors. ANOVA was used 
to test change over time in reported net OS benefit. Testing for correlations was performed using the Pearson correlation coefficient. The sensitivity and specificity of various cutoff values of NHB score for predicting NCCN Guidelines citation were calculated. The corresponding receiver operating characteristic (ROC) curve was generated using SAS 9.3 (SAS Institute, Cary, NC). All tests of significance ( $P$ values) were 2 -sided. Statistical significance was represented by $P<.05$. Analysis was performed using SAS 9.3.

\section{Results}

\section{Evolution of mCRC Trials}

We identified $114 \mathrm{mCRC}$ trials that met our inclusion criteria (Figure 1). A trend was seen toward higher NHB score for second-line trials compared with first-line trials (median revised NHB score, 10.00 vs $3.41 ; P=.07$ ). In the 109 trials that reported OS data, improvements in the median net OS benefit are shown in Figure 2. No significant change occurred in net OS benefit over time $(P=.76)$. Despite this lack of significant change, the average mOS reported in all trials has increased over the decades regardless of line of therapy: average mOS for first-line

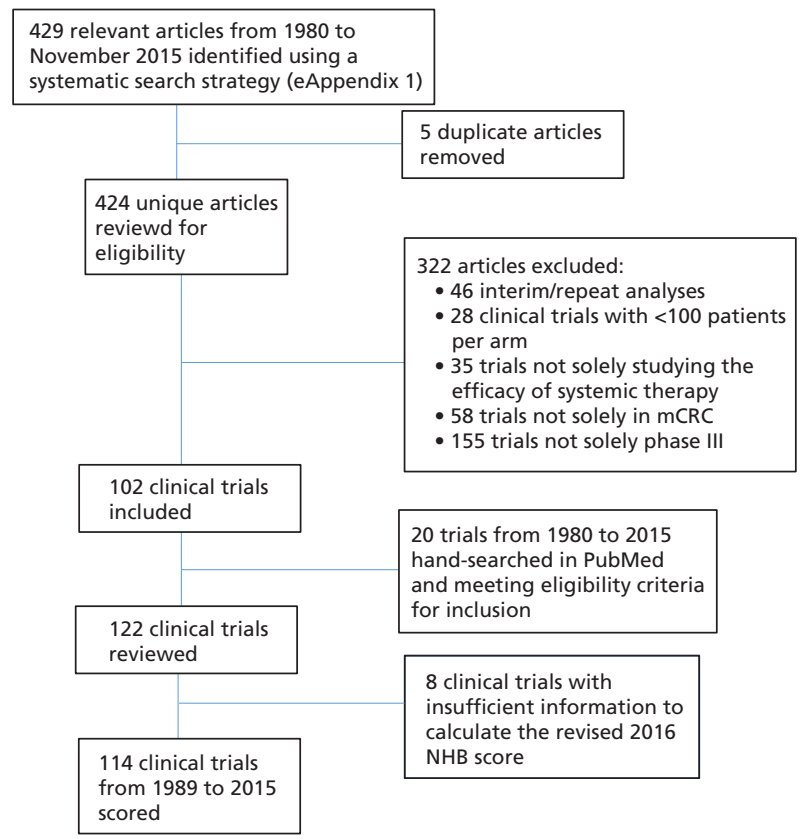

Figure 1. mCRC clinical trials analyzed with the 2016 ASCO Value Framework.

Abbreviations: $m C R C$, metastatic colorectal cancer; NHB, net health benefit.

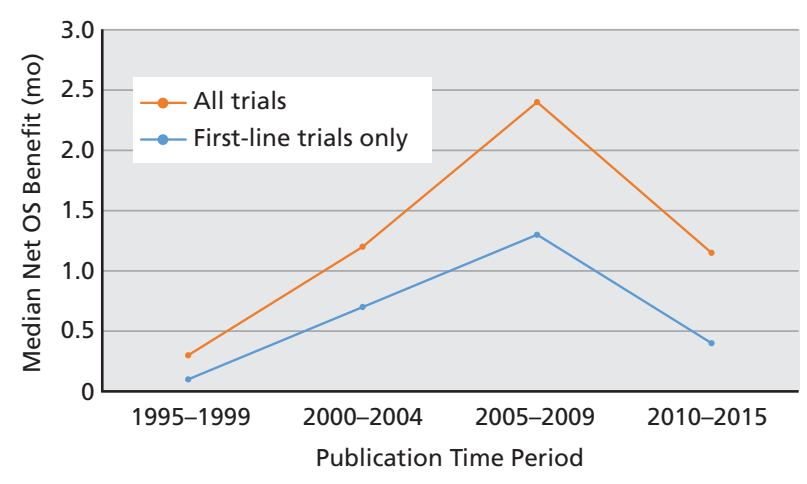

Figure 2. Median net overall survival (OS) benefit over time reported in all trials (orange line) and first-line trials only (blue line).

Note: The time period from 1980 to 1995 had no trials meeting inclusion criteria that reported OS results.

trials gradually increased from 11.6 months in the 1995-1999 strata, to 15.5 months in the 2000-2004 period, to 19.2 months in the 2005-2009 period, to 20.7 months in the 2010-2015 strata (Table 1).

\section{Application of the ASCO Value Framework}

Median revised $2016 \mathrm{NHB}$ score was 4.6 (range, -30 to 43.5; Table 1). When noninferiority trials were excluded, median NHB score was 5.1. A total of $12 \%$ of the studies obtained bonus points for improvement in cancer-related symptoms, tail of the curve, or QoL (Table 1). No studies received treatment-free interval bonus points. A total of $17 \%$ of the studies reported a statistically significant clinical end point used to generate the NHB score, with OS being the clinical end point used for scoring in most cases. Approximately half of the studies $(48 \%)$ were cited by the NCCN Guidelines.

In contrast, the median NHB score using the original 2015 framework was 16, with approximately half of the studies $(48 \%)$ achieving a NHB score of 16 (Table 1). NHB scores were normally distributed using the revised framework, unlike the original framework (Figure 3). The revised framework demonstrated moderate correlation with the original framework (Pearson correlation coefficient, 0.64; $P<.0001)$.

Further analyses were conducted using the revised 2016 NHB scores (Table 2). The median year of publication for the studies analyzed was 2008. Analysis of the NHB of trials published before and after 2008 showed no significant change in $\operatorname{NHB}(P=.3$; Table 2). Figure 4 depicts the median NHB score over time. When the period from 1989 to 2015 was 
Ezeife et al

\begin{tabular}{|c|c|c|}
\hline & $\begin{array}{c}\text { Revised NHB } \\
n(\%) \\
(\mathrm{N}=114)\end{array}$ & $\begin{array}{c}\text { Original NHB } \\
n(\%) \\
(\mathrm{N}=113)\end{array}$ \\
\hline Year, median (range) & $2008(1989-2015)$ & $2008(1989-2015)$ \\
\hline Sample size of study, median (range) & $485(200-2,397)$ & $476(200-2,135)$ \\
\hline NHB & $\begin{array}{l}\text { Mean: } 4.2 \\
\text { Median (range): } 4.6 \text { (-30 to } 43.5 \text { ) }\end{array}$ & $\begin{array}{l}\text { Mean: } 12.2 \\
\text { Median (range): } 16 \text { ( }-10 \text { to } 32 \text { ) }\end{array}$ \\
\hline Toxicity score & $\begin{array}{l}\text { Mean: }-2.4 \\
\text { Median (range): }-1.6 \text { (-46.8 to 9.2) }\end{array}$ & $\begin{array}{l}\text { Mean: }-1.02 \\
\text { Median (range): } 0 \text { ( }-20 \text { to } 0)\end{array}$ \\
\hline \multicolumn{3}{|l|}{ Original NHB score, group } \\
\hline$<16$ & N/A & $38(35)$ \\
\hline 16 & N/A & $51(48)$ \\
\hline$>16$ & N/A & $18(17)$ \\
\hline NHB excluding noninferiority trials $(n=101)$ & $\begin{array}{l}\text { Mean: } 4.7 \\
\text { Median (range): } 5.1 \text { (-30 to } 43.5 \text { ) }\end{array}$ & \\
\hline \multicolumn{3}{|l|}{ Line of therapy $(n)$} \\
\hline First-line trials & 85 & \\
\hline \multirow[t]{2}{*}{ Second- and third-line trials } & 29 & \\
\hline & 2000-2004 & 2005-2009 \\
\hline Average OS by strata, all trials (mo) & 11.4 & 17.3 \\
\hline First-line trials & 11.6 & 19.2 \\
\hline Second- and third-line trials & 6.8 & 12.6 \\
\hline Statistically significant studies & $18(17 \%)$ & \\
\hline Studies cited in NCCN Guidelines & $55(48 \%)$ & \\
\hline \multicolumn{3}{|l|}{ Total bonus points } \\
\hline 0 & $100(87.72 \%)$ & \\
\hline 10 & $13(11.40 \%)$ & \\
\hline 20 & $1(0.88 \%)$ & \\
\hline Studies that received palliative bonus points & $2(1.8 \%)$ & \\
\hline $\begin{array}{l}\text { Studies that received treatment-free interval } \\
\text { or tail-of-the-curve bonus points }\end{array}$ & 0 & \\
\hline Studies that received QoL bonus points & $12(10.5 \%)$ & \\
\hline Impact factor, median (range) & $18(2.0-55)$ & \\
\hline \multicolumn{3}{|l|}{ Impact factor by group, median (mean) } \\
\hline $0-9.99$ & $35(31)$ & \\
\hline 10-19.99 & $50(44)$ & \\
\hline$\geq 20$ & $29(25)$ & \\
\hline
\end{tabular}

Abbreviations: N/A, not applicable; NHB, net health benefit; OS, overall survival; QoL, quality of life.

divided into 5-year groups, no significant change was seen in median NHB score ( $P=.27$; data not shown). The median journal impact factor for the studies included was 18 . NHB score had a weak correlation with journal impact factor (Pearson correlation coefficient, $0.25 ; P=.006$ ). Trials with statistically significant results had significantly higher NHB scores (median NHB score, 21.6 vs 2.9; $P<.0001$; Table 2 ). When NHB score was stratified by the median of 4.6 , statistically significant studies tended to have higher
NHB scores $(89 \%$ vs $11 \%$ of statistically significant studies had a NHB score $\geq 4.6 ; P=.00002$; Table 3 ).

Clinical trials cited in NCCN Guidelines for CRC had higher NHB scores than those not cited (median NHB score, 8.0 vs $0.3 ; P=.02$; Table 2). When stratified by the median revised NHB score, studies cited in NCCN Guidelines tended to have higher NHB scores (65\% vs 35\%; $P<.001$; Table 3 ). Figure 5 shows the corresponding ROC curve for NHB score predicting NCCN Guidelines citation 
A

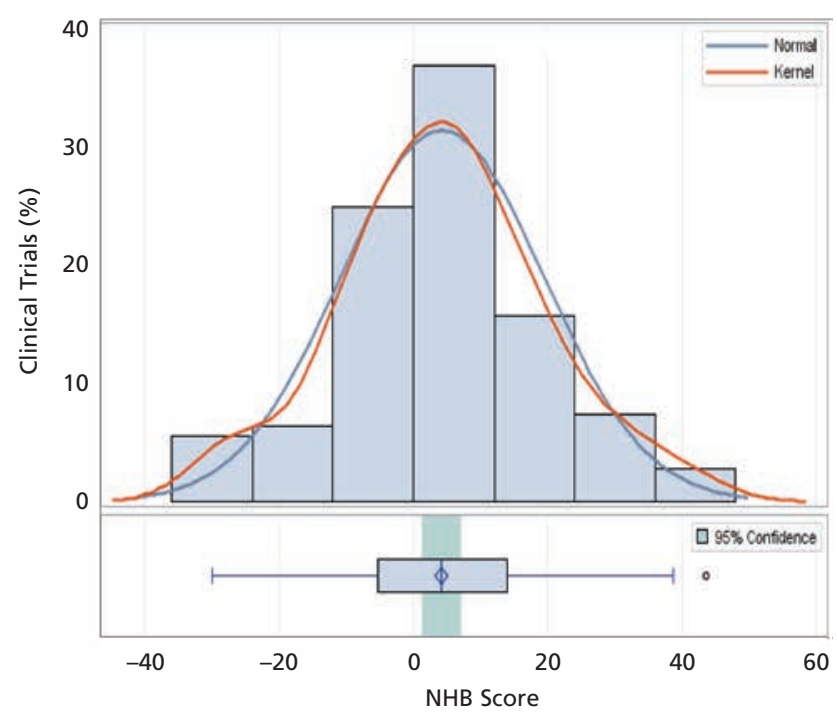

B

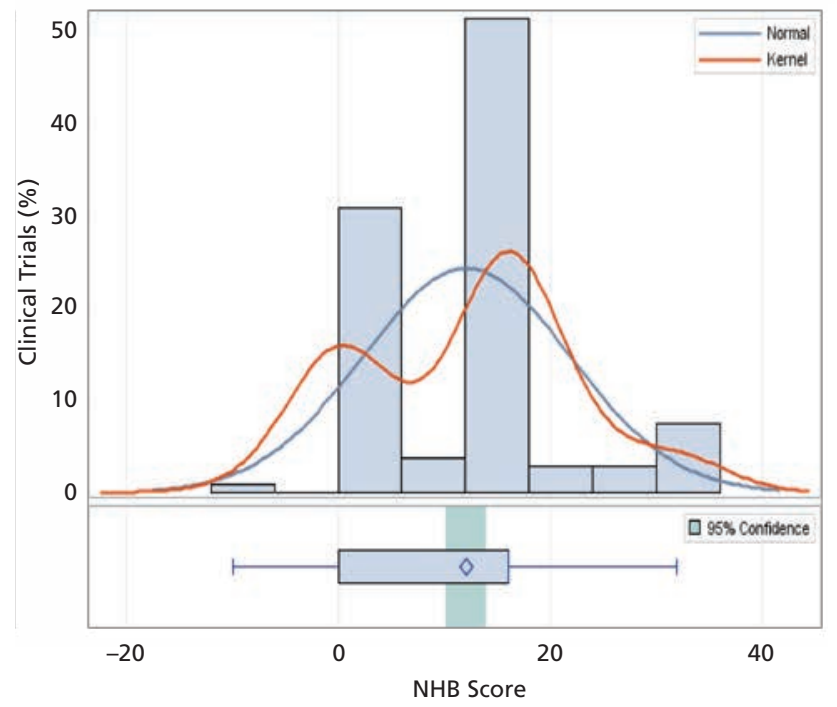

Figure 3. Histogram distribution curves of (A) revised NHB and (B) original NHB scores with $95 \% \mathrm{Cl}$ for mean. Abbreviation: NHB, net health benefit.

(AUC, 0.64). There was no association between revised NHB score and trial sample size or toxicity score. When multivariate linear regression analysis was performed, the only significant predictor of a high revised NHB score was a statistically significant published outcome (point estimate, 15.5; 95\% CI, 8.1-23; $P<.0001$ ). This finding was independent of other variables, such as NCCN citation, journal impact factor, publication year, and trial sample size.

Table 4 shows the NHB score and the associated incremental cost of $4 \mathrm{mCRC}$ regimens for which information was publically available. The bevacizum$\mathrm{ab} /$ capecitabine regimen achieved the highest NHB score; however, it also had the highest incremental cost and ICER. Conversely, the regimen with the lowest incremental cost (regorafenib) did not have the highest NHB score.

\section{Discussion}

Our analysis uses the ASCO Value Framework to describe the incremental improvements of new systemic therapies established by phase III mCRC clinical trials. The maximum possible NHB score is 130 in the original framework. Due to the adoption of a continuous scale for assessing NHB in the revised 2016 framework, the maximum possible NHB score is undefined. Median revised NHB score for phase
III mCRC clinical trials was 4.6, establishing a bar for further progress. Because the revised framework prioritizes the hazard ratio (HR) and uses more elaborate toxicity scoring, high NHB scores are difficult to achieve in the mCRC setting. Additionally, proportionately larger sample sizes are required in patients with $\mathrm{mCRC}$ in order to achieve impressive HRs for the same improvement in mOS, a phenomenon that has been previously described. ${ }^{12}$ We observed a trend toward higher NHB scores in second- and third-line trials compared with first-line trials ( 10.00 vs 3.41 ; $P=.07$ ), likely due to the increased number of targeted therapies achieving high NHB scores in the second- and third-line setting. In later lines of therapy, patients may prioritize QoL and OS over toxicity, a reality that is not fully captured by the NHB score.

Most studies either did not collect or found no improvement in cancer-related symptoms, QoL, or treatment-free interval with the experimental regimen. Our study found that only 33\% of the mCRC clinical trials incorporated patient-reported outcomes (PROs). Treatment-related toxicity, in the absence of advancements or PRO reporting, contributed to the low revised NHB scores. Prior data have highlighted the importance of PROs in clinical trials to support patient-centered decision-making in CRC and other cancers. ${ }^{13}$ Integration and enhancement of 
Ezeife et al

\begin{tabular}{|c|c|c|}
\hline & \multicolumn{2}{|c|}{ Revised NHB Score } \\
\hline & $\begin{array}{l}\text { Mean NHB } \\
\text { (median) }\end{array}$ & $\begin{array}{l}P \text { Value } \\
\text { (t test) }\end{array}$ \\
\hline Year class & & .3 \\
\hline Before $2008(n=54)$ & $5.7(5.2)$ & \\
\hline 2008 and after $(n=60)$ & $2.9(3.7)$ & \\
\hline Impact factor, class & & .006 \\
\hline$<18$ & $-1.5(-1.7)$ & \\
\hline$\geq 18$ & $6.8(7.0)$ & \\
\hline Impact factor, group & $10.0(10.0)$ & .008 \\
\hline 0-9.99 & $-1.5(-1.7)$ & \\
\hline 10-19.99 & $5.0(5.9)$ & \\
\hline$\geq 20$ & $-1.5(-1.7)$ & \\
\hline Sample size & & .1 \\
\hline$<485$ patients (median) & $2.0(2.6)$ & \\
\hline$\geq 485$ patients & $6.5(5.3)$ & \\
\hline \multicolumn{3}{|l|}{ Significant studies } \\
\hline Statistically significant & $18.8(21.6)$ & $<.0001$ \\
\hline Nonsignificant & $1.5(2.9)$ & \\
\hline Cited in NCCN, n & & .02 \\
\hline Yes & $7.5(8.0)$ & \\
\hline No & $1.2(0.3)$ & \\
\hline Total bonus points & & .0001 \\
\hline 0 & $2.3(3.3)$ & \\
\hline$>0$ & $18.4(19.4)$ & \\
\hline Toxicity score & & .8 \\
\hline$<-1.7$ & $4.5(6.6)$ & \\
\hline$\geq-1.7$ & $3.9(3.4)$ & \\
\hline
\end{tabular}

Abbreviation: NHB, net health benefit.

PROs in clinical trials can improve the ASCO Value Framework's ability to assess clinical trial data.

The ASCO Value Framework was designed to facilitate clinical decision-making for the oncologist and patient, and can only be applied to phase III clinical trials. Although randomized phase II trials have the potential to be practice-changing in the era of biomarker-driven trials in molecular subsets, they cannot be assessed using the current ASCO framework.

Cost of new therapies is critical in assessing the added value of a drug relative to its NHB score. We compared the NHB score to the incremental cost of 28 days of therapy for $4 \mathrm{mCRC}$ chemotherapy regimens. The AVEX trial, demonstrating the superiority of bevacizumab/capecitabine over capecitabine alone for first-line therapy of mCRC, was the only trial that

\begin{tabular}{|cccc|}
\hline Table 3. NHB Score and Association With Study \\
Significance and NCCN Citation \\
$\begin{array}{cccc}\text { Nonsignificant } \\
\text { Study }\end{array}$ & $\begin{array}{c}\text { Statistically } \\
\text { Significant } \\
\text { Study }\end{array}$ & $\begin{array}{c}\text { P Value } \\
\text { (Fisher exact test) }\end{array}$ \\
\hline NHB $<4.6$ & $54(57 \%)$ & $2(11 \%)$ & .0002 \\
NHB $\geq 4.6$ & $40(43 \%)$ & $17(89 \%)$ & $\begin{array}{c}P \text { Value } \\
\left(X^{2} \text { test) }\right.\end{array}$ \\
\hline & NCCN Not Cited & NCCN Cited & .001 \\
\cline { 1 - 3 } NHB $<4.6$ & $38(64 \%)$ & $19(35 \%)$ & \\
NHB $\geq 4.6$ & $21(36 \%)$ & $36(65 \%)$ & \\
\hline
\end{tabular}

Abbreviation: NHB, net health benefit.

earned tail-of-the-curve bonus points. ${ }^{14}$ Of the 4 trials, it had the highest NHB score. This prompts the question of whether the tail-of-the-curve bonus points and impressive HR for OS (HR, 0.79; 95\% CI, 0.57-1.09; $P=.18$ ) justifies the nearly $\$ 40,000$ USD incremental cost. ${ }^{11}$ This clinical trial is also an example of how the ASCO framework prioritizes HRs for OS over PFS, regardless of statistical significance.

In contrast, in the treatment-refractory setting, the NHB for regorafenib versus placebo was 10 , driven by the poor outcome in the control arm. The incremental cost was substantially lower. However, because of the toxicity and relatively small incremental benefit, the ICER was $\$ 141,403$ per quality-adjusted life year. ${ }^{11}$ Ultimately, these incremental cost data highlight that the ASCO framework cannot be used to perform cross-trial comparisons, because the NHB score depends on the incremental differences in outcomes between the experimental and control arms. The data also underscore the individual nature of the decision made by the oncologist and patient regarding whether a new therapeutic agent's benefits outweigh its harms.

Our findings confirm that, unlike the original 2015 framework, the revised 2016 NHB scores were normally distributed for mCRC trials. The use of HRs and more precise toxicity scoring are contributors to the more robust metric of the revised ASCO value assessment tool. We applaud the inclusion of clinical outcomes important to patients with advanced cancer in the revised ASCO Value Framework: OS, QoL, and symptom palliation. ${ }^{12,15}$ However, the ASCO framework weights the OS end point more heavily than PFS or response rate, regardless of whether there is statistical significance achieved in any of the end points. Our data indicate an association between a NHB score and published statisti- 
Evolution of mCRC Trials: Value

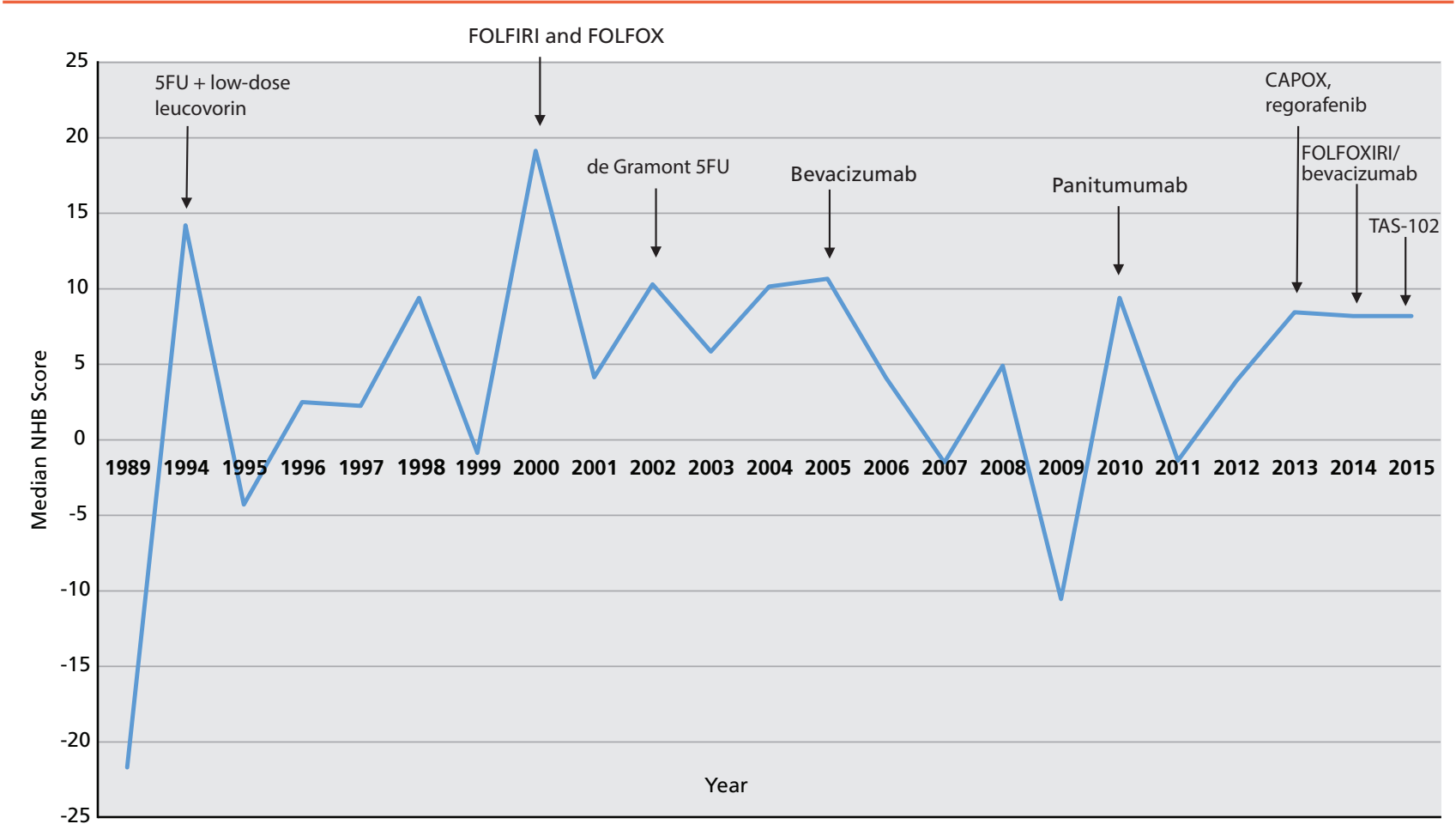

Figure 4. Median net health benefit (NHB) score per year. Pivotal trials that studied new practice-changing therapies are highlighted in the appropriate year that the trial was published (eg, 5-fluorouracil [5FU] + low-dose leucovorin: 1994; FOLFIRI: 2000; panitumumab: 2010). These pivotal trials resulted in peaks in the NHB score for the given year.

cal significance. Prior criticism of the ASCO Value Framework has acknowledged its inability to assess the methodological strength of evidence. ${ }^{16}$ Including a consideration of statistical significance in the clini-

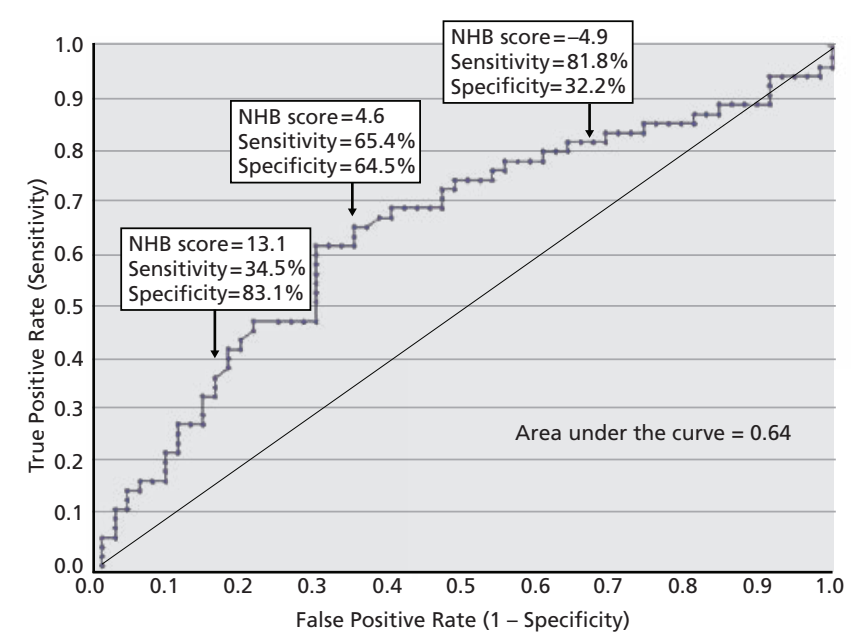

Figure 5. ROC curve of NHB score and NCCN citation. ROC curve depicts the NHB score and corresponding sensitivity and specificity of $\mathrm{NHB}$ score predicting NCCN citation, a surrogate for a practice-changing trial.

Abbreviations: NHB, net health benefit; ROC, receiver operating characteristic. cal benefit component of the NHB score can allow for stronger evaluation of efficacy.

Recently, several other value frameworks have been developed for use in cancer care. ESMO designed a tool to evaluate new cancer treatments for drug approval based on their magnitude of clinical benefit, toxicity, and QoL. ${ }^{17}$ The NCCN Evidence Blocks is a patient-oriented value formula that considers affordability (including drug cost, required supportive care, infusions, toxicity monitoring and management, and probability of care being delivered in the hospital), drug efficacy and safety, quality and quantity of clinical data, and consistency of clinical data. ${ }^{18}$ In Canada, the pCODR program provides evidence-based drug funding recommendations by considering overall clinical benefit (including effectiveness, safety, burden of illness, and need), alignment with patient values, cost-effectiveness, and feasibility of adoption into health systems. These alternative value assessment tools have been previously critiqued and compared with one another. $6,16,19,20$ We support the inclusion of QoL in the revised ASCO Value Framework because it is an important factor for patients with advanced cancer. Addition- 
Ezeife et al

\begin{tabular}{|c|c|c|c|c|}
\hline $\begin{array}{l}\text { Clinical } \\
\text { Trial }\end{array}$ & Regimen & $\begin{array}{l}\text { Revised } \\
\text { NHB } \\
\text { Score }\end{array}$ & $\begin{array}{c}\text { Incremental } \\
\text { Cost, \$USD } \\
\text { (Per 28-Day } \\
\text { Cycle) }\end{array}$ & $\begin{array}{c}\text { ICER } \\
\text { (\$USD/QALY) }\end{array}$ \\
\hline PRIME & $\begin{array}{l}\text { FOLFOX/ } \\
\text { panitumumab } \\
\text { (first line) }\end{array}$ & 5.3 & $\$ 32,894$ & $N A^{b}$ \\
\hline AVEX & $\begin{array}{l}\text { Bevacizumab/ } \\
\text { capecitabine } \\
\text { (first line) }\end{array}$ & 38.43 & $\$ 39,777$ & $\$ 159,704$ \\
\hline CORRECT & $\begin{array}{l}\text { Regorafenib } \\
\text { (second line or } \\
\text { later) }\end{array}$ & 10 & $\$ 11,745$ & $\$ 141,403$ \\
\hline VELOUR & $\begin{array}{l}\text { FOLFIRI/ } \\
\text { aflibercept } \\
\text { (second line) }\end{array}$ & 16.2 & $\$ 12,802$ & $\$ 105,278$ \\
\hline
\end{tabular}

Abbreviations: NHB, net health benefit; ICER, incremental costeffectiveness ratio; NA, not available; QALY, quality-adjusted life years.

alncremental cost represents the increased incremental cost of the experimental regimen compared with the control regimen in the associated clinical trial.

bICER was not available for the PRIME clinical trial.

ally, inclusion of robust cost-effectiveness measures can expand the representation of a drug's value from the individual patient level to its impact on society, especially in third-party (insured) and universal healthcare systems.

Some limitations of this study should be noted. In isolation, the numerical NHB score does not fully depict the value of a drug. Only 4 regimens had incremental cost data available. It should be noted that the incremental cost data presented is the manufacturer's listed price upon submitting for funding in Canada, and therefore temporal and geographic variations will exist. Ultimately, using the ASCO framework in clinical decisions does require consideration of the cost of a drug in addition to the
NHB. We evaluated the ASCO Value Framework of mCRC trials to explore the association of the NHB score with high impact, practice-changing trials. The ASCO Value Framework was not designed for cross-trial comparison. It should also be noted that our analysis was performed using the advanced disease framework for metastatic disease. Different results will likely be obtained in the curative cancer setting using the adjuvant framework. Application of the ASCO framework to other disease sites will likely yield different findings as well.

\section{Conclusions}

The present analyses raise questions regarding the value of new mCRC treatments. With the advent of targeted therapy and immunotherapy, cancer treatments can reach $\$ 100,000$ USD per month. ${ }^{19}$ The ASCO Value Framework applied in the mCRC setting demonstrates a median NHB score of only 4.6. To our knowledge, this is the first systematic application of the ASCO Value Framework in advanced cancer. In an evolving era with the potential for more precise biomarker-driven therapy, the structured ASCO Value Framework has limited utility in capturing the benefits on an individual patient level. We suggest that future iterations of the ASCO framework implement consideration of statistical significance to efficacy outcomes in order to better identify high-value studies. In light of the more complex toxicity grading in the revised framework, we commend ASCO's efforts to convert the framework to a user-friendly software application. ${ }^{8}$ Ultimately, incorporating a standard, easy-to-use, value assessment tool can help patients, clinicians, and policymakers practice value-based cancer care.

\section{References}

1. Heinemann $V$, von Weikersthal LF, Decker $T$, et al. FOLFIRI plus cetuximab versus FOLFIRI plus bevacizumab as first-line treatment for patients with metastatic colorectal cancer (FIRE-3): a randomised, openlabel, phase 3 trial. Lancet Oncol 2014;15:1065-1075.

2. Douillard JY, Cunningham D, Roth AD, et al. Irinotecan combined with fluorouracil compared with fluorouracil alone as first-line treatment for metastatic colorectal cancer: a multicentre randomised trial. Lancet 2000;355:1041-1047.

3. Saltz LB, Cox JV, Blanke C, et al. Irinotecan plus fluorouracil and leucovorin for metastatic colorectal cancer. Irinotecan Study Group. N Engl J Med 2000;343:905-914.

4. Nardi EA, Wolfson JA, Rosen ST, et al. Value, access, and cost of cancer care delivery at academic cancer centers. J Natl Compr Canc Netw 2016;14:837-847.

5. Douillard JY, Siena S, Cassidy J, et al. Final results from PRIME: randomized phase III study of panitumumab with FOLFOX4 for first-line treatment of metastatic colorectal cancer. Ann Oncol 2014;25:1346-1355.

6. Schnipper LE, Bastian A. New frameworks to assess value of cancer care: strengths and limitations. Oncologist 2016;21:654-658.

7. Schnipper LE, Davidson NE, Wollins DS, et al. American Society of Clinical Oncology statement: a conceptual framework to assess the value of cancer treatment options. J Clin Oncol 2015;33:2563-2577.

8. Schnipper LE, Davidson NE, Wollins DS, et al. Updating the American Society of Clinical Oncology value framework: revisions and reflections in response to comments received. J Clin Oncol 2016;34:2925-2934.

9. Benson AB III, Venook AP, Bekaii-Saab T, et al. NCCN Clinical Practice Guidelines in Oncology: Colon Cancer, Version 1.2016. Accessed July 16, 2016. To view the most recent version of these guidelines, visit NCCN.org. 
10. Benson AB III, Venook AP, Bekaii-Saab T, et al. NCCN Clinical Practice Guidelines in Oncology: Colon Cancer, Version 2.2016. Accessed July 16, 2016. To view the most recent version of these guidelines, visit NCCN.org.

11. CADTH pan-Canadian Oncology Drug Review. CADTH Web site Available at: https://cadth.ca/pcodr. Accessed February 26, 2017.

12. Sargent DJ, Hayes DF. Assessing the measure of a new drug: is survival the only thing that matters? J Clin Oncol 2008;26:1922-1923.

13. Rees JR, Whale K, Fish D, et al. Patient-reported outcomes in randomised controlled trials of colorectal cancer: an analysis determining the availability of robust data to inform clinical decision-making. J Cancer Res Clin Oncol 2015;141:2181-2192.

14. Cunningham D, Lang I, Marcuello E, et al. Bevacizumab plus capecitabine versus capecitabine alone in elderly patients with previously untreated metastatic colorectal cancer (AVEX): an open-label, randomised phase 3 trial. Lancet Oncol 2013;14:1077-1085.

15. Burzykowski T, Buyse M, Piccart-Gebhart MJ, et al. Evaluation of tumor response, disease control, progression-free survival, and time to progression as potential surrogate end points in metastatic breast cancer. J Clin Oncol 2008;26:1987-1992.

16. Cheung MC, Sabharwal M, Chambers A, et al. Multiple dimensions of value: evaluative frameworks for new cancer therapies. J Clin Oncol 2016;34:1428-1429.

17. Cherny NI, Sullivan R, Dafni U, et al. A standardised, generic, validated approach to stratify the magnitude of clinical benefit that can be anticipated from anti-cancer therapies: the European Society for Medical Oncology Magnitude of Clinical Benefit Scale (ESMO-MCBS). Ann Oncol 2015;26:1547-1573

18. NCCN Clinical Practice Guidelines in Oncology (NCCN Guidelines $\left.{ }^{\circledR}\right)$ with NCCN Evidence Blocks ${ }^{\mathrm{TM}}$. Available at: https://www.nccn.org/ evidenceblocks/. Accessed July 18, 2016.

19. Young RC. Value-based cancer care. N Engl J Med 2015;373:2593-2595.

20. Angelis A, Kanavos P. Critique of the American Society of Clinical Oncology value assessment framework for cancer treatments: putting methodologic robustness first. J Clin Oncol 2016;34:2935-2936.

\section{Redefining Quality Measurement in Oncology}

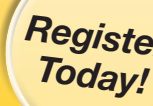

Monday, September 25, 2017

9:00 AM - 3:00 PM

(Registration and Breakfast begin at 8:00 AM)

The National Press Club | Washington, DC
This policy summit will be a forum for stakeholders to discuss the challenges and opportunities for measuring quality care in oncology. The summit will examine the past and current efforts toward developing outcomes-based oncology quality measures, discuss the value of those measures, the role these measures play in payment reform and whether patient care and the patient experience has improved as a result. The impact of emerging payment models on quality cancer care will be considered.
Agenda* topics include:

- The Quality Measure Landscape in Oncology

- Incorporating Patient Perspectives in Quality Measurement of Cancer Care

- Panel Discussion: Measuring Quality in Oncology: Challenges, Lessons Learned, and the Way Forward

- Highlighting Best Practices in Quality Measurement and Reporting in Cancer Care

- Panel Discussion: The Future of Quality Reporting in Cancer Care

${ }^{*}$ Agenda subject to change

\section{NCCN.org/policy}

